${ }^{1}$ Institut für Arbeitsmedizin, Charité - Universitätsmedizin Berlin, Zentrum für Humanund Gesundheitswissenschaften, Freie Universität \& Humboldt-Universität zu Berlin ${ }^{2}$ Institut für Medizinische Virologie, Klinikum der Johann Wolfgang Goethe-Universität Frankfurt am Main

${ }^{3}$ Betriebsärztlicher Dienst, Klinikum der Johann Wolfgang Goethe-Universität Frankfurt am Main

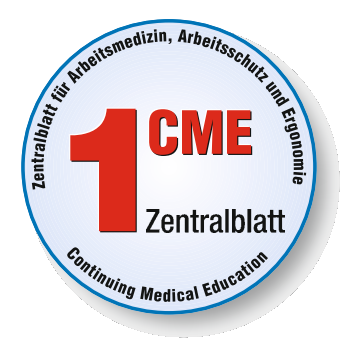

\title{
Die Bedeutung der aviären Influenza für das Gesundheitssystem
}

Miriam Molliné ${ }^{1}$, Lea Gerischer ${ }^{1}$, Ralph Fricke ${ }^{1}$, Carolin Kreiter $^{1}$, David Quarcoo ${ }^{1}$, Holger F. Rabenau ${ }^{2}$, Sabine Wicker ${ }^{2,3}$, David A. Groneberg ${ }^{1}$

M. Molliné, L. Gerischer, R. Fricke, C. Kreiter, D. Quarcoo, H.F. Rabenau, S. Wicker, D.A. Groneberg: Die Bedeutung der aviären Influenza für das Gesundheitssystem. Zbl Arbeitsmed 58 (2008) 204-214

Schlüsselwörter:

Aviäre Influenza - Vogelgrippe - Influenza-Viren - Pandemie

Zusammenfassung:

Die aviäre Influenza ist eine Viruskrankheit, die insbesondere Vögel befällt. Die auslösenden Erreger der Erkrankung sind Influenza-Viren. Die Erkrankung ist weltweit bekannt und wurde erstmals 1878 in Italien beschrieben. Influenza-Viren gehören zur Gruppe der Orthomyxoviridae. Das Influenza-A-Virus H5N1 gilt seit seiner sprunghaften Ausbreitung unter Geflügel im Jahre 2003 als möglicher Auslöser einer neuen Pandemie.

H5N1 ist humanpathogen, es besteht keine Immunität beim Menschen und es ist hochpathogen mit einer Mortalitätsrate von über $60 \%$. Die wesentliche, bisher fehlende Eigenschaft des Virus um eine Pandemie auszulösen, ist die mangelnde effektive Übertragung von Mensch zu Mensch. Der vorliegende Übersichtsartikel befasst sich mit der Bedeutung der aviären Influenza für das Gesundheitssystem.

\section{The impact of avian influenza on health care system}

M. Molliné, L. Gerischer, R. Fricke, C. Kreiter, D. Quarcoo, H.F. Rabenau, S. Wicker, D.A. Groneberg: The impact of avian influenza on health care system. Zbl Arbeitsmed 58 (2008) 204-214

Key words:

avian influenza - bird flu - influenza virus - pandemic

\section{Summary:}

Avian influenza is a viral transmitted disease that mainly occurs in birds. First described in 1878 in Italy the disease can be found worldwide. The causing agent belongs to the family of orthomyxoviridae. Since its enormous spread among poultry in 2003, the influenza virus H5N1 is heralded as a possible agent of a new pandemic. H5N1 is pathogen to humans with a mortality rate of over $60 \%$. The main reason that this virus has not caused a pandemic yet is its lack to propagate freely between humans. The current review explores the impact of avian influenza on the health system.

\footnotetext{
Anschrift der Autoren:

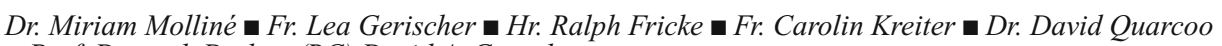

- Prof. Dr. med. Dr. h.c. (RC) David A. Groneberg

Institut für Arbeitsmedizin, Charité - Universitätsmedizin Berlin, Zentrum für Human- und Gesundheitswissenschaften,

Freie Universität \& Humboldt-Universität zu Berlin

Thielallee 67-73 14195 Berlin

Prof. Dr. Holger F. Rabenau Dr. Sabine Wicker

Institut für Medizinische Virologie, Klinikum der Johann Wolfgang Goethe-Universität Frankfurt am Main

Paul-Ehrlich-Str. 40 — 60596 Frankfurt am Main

Dr. Sabine Wicker

Betriebsärztlicher Dienst, Klinikum der Johann Wolfgang Goethe-Universität Frankfurt am Main

Theodor-Stern-Kai 7 - 60590 Frankfurt am Main
} 


\section{Einleitung}

Die aviäre Influenza ist eine Viruskrankheit, die Wildvögel - vor allem Wasservögel - aber auch Ziervögel und Geflügel in der Tierhaltung, hier vor allem Hühner sowie Puten, befällt. Die auslösenden Erreger der auch als Geflügelpest oder Vogelgrippe bezeichneten Erkrankung sind Influenza-A-Viren. Die Erkrankung ist weltweit verbreitet und wurde erstmals 1878 in Italien beschrieben (Allwinn \& Doerr 2005). Influenza-Viren gehören zur Gruppe der Orthomyxoviridae, einer Gruppe von Einzelstrang-RNA-Viren mit segmentierten Genomen. Man unterscheidet Influenza-A-, -B- und -C-Viren. Influenza-B-Viren kommen nur beim Menschen vor, Influenza-C-Viren spielen dagegen beim Menschen nur eine untergeordnete Rolle (Allwinn \& Doerr 2005). Aufgrund des großen natürlich vorhandenen Virusreservoirs von Influenza-A-Viren in Tieren, z.B. in Wasservögeln, ist die Eradikation des Virus erschwert. Es wird davon ausgegangen, dass nur Influenza-A-Viren in der Lage sind, eine Pandemie auszulösen (Perez et al. 2005). Die Unterscheidung der Influenza-A-Viren in Subtypen erfolgt anhand der virusspezifischen Oberflächenproteine Hämagglutinin $(\mathrm{H})$ und Neuraminidase (N). Vom Hämagglutinin sind 16 Subtypen bekannt, von der Neuraminidase 9 Subtypen (Wong \& Yuen 2006). Somit sind theoretisch insgesamt 144 Kombinationen dieser beiden Proteine möglich. Verantwortlich für die schweren Ausbrüche der Geflügelpest sind hochpathogene aviäre Influenzastämme der Subtypen H5 und H7 (Allwinn \& Doerr 2005)

Im Jahr 1997 wurde das Vogelgrippe Virus H5N1 erstmalig als für den Menschen gefährlich eingestuft. Bei einem Geflügelpestausbruch in Hong Kong wurden 18 Menschen infiziert, sechs starben (Juckett 2006). Man ging davon aus, dass die Gefahr gebannt sei, nachdem die gesamten Geflügelbestände in Hong Kong geschlachtet waren. Jedoch wurden im Jahr 2003 neue Fälle von Vogelgrippe in Hong Kong gemeldet und kurz darauf kamen Ausbrüche von Vogelgrippe in Thailand, Vietnam, Indonesien, Kambodscha und China hinzu
(Juckett 2006). All diese Ausbrüche wurden durch das H5N1-Virus verursacht. Im Jahr 2006 verbreitete sich das Virus über Wildvögel bis in die Türkei und Russland und erreichte schließlich Europa, darunter auch Deutschland, sowie einige afrikanische Länder wie beispielsweise Nigeria. Bis zum 15. April 2008 wurden 380 menschliche Fälle von aviärer Influenza aus 14 Ländern gemeldet, 240 Personen waren an der Krankheit verstorben (WHO 2008). Die meisten von ihnen hatten engen Kontakt mit infizierten Vögeln gehabt (Juckett 2006). Bislang wurde die Übertragbarkeit auf ca. 90 Vogelarten und mehr als 10 Säugetierarten nachgewiesen - u.a. auf Katzen, Tiger, Leoparden, Steinmarder und Schweine. Obwohl H5N1 nicht das einzige Influenza-A-Virus ist, welches in den letzten Jahren Ausbrüche von Vogelgrippe verursacht hat, spricht vieles dafür, dass es ein möglicher Auslöser der nächsten Influenza-Pandemie sein könnte. Das Virus besitzt drei von vier notwendigen Eigenschaften um eine Pandemie auszulösen: Es ist humanpathogen, beim Menschen besteht keine Immunität und es ist hochpathogen mit einer Mortalitätsrate von über $60 \%$ (WHO 2008). Eine wesentliche Eigenschaft, die dem Virus noch fehlt, ist eine effiziente Übertragbarkeit von Mensch zu Mensch. Das Virus benötigt u.U. nur wenige Mutationen, um diese Eigenschaft zu erlangen (Bartlett 2006).

In dieser Übersichtsarbeit wird die Bedeutung der Vogelgrippe für das öffentliche Gesundheitswesen auf verschiedenen Ebenen dargestellt. Dabei wird besonders auf die Pandemiegefahr eingegangen. Auf internationaler Ebene werden die aktuellen Pläne und Empfehlungen der Weltgesundheitsorganisation (WHO) analysiert. Überregional wird sowohl auf die Lage in der Europäischen Union und insbesondere der Bundesrepublik Deutschland als auch auf die Lage in der asiatisch-pazifischen Region eingegangen, in welcher bis jetzt die meisten Fälle von H5N1-Infektionen bei Menschen aufgetreten sind.

\section{Globale Situation und Rolle der WHO}

Seitdem sich das H5N1-Influenza A-Virus im Jahr 2003 unter Geflügel ausgebreitet hat, hat die Weltgesundheitsorganisation (WHO) auf die Gefahr einer erneuten Pandemie hingewiesen und alle Länder dazu aufgerufen, sich auf die damit verbundenen Aufgaben vorzubereiten (Normile 2006). Die WHO hat im Jahr 2005 eine neue Version ihres Influenza-Plans veröffentlicht und dazu eine Prüfliste bereit gestellt, mit deren Hilfe nationale Influenza-Pläne auf Vollständigkeit überprüft werden können (WHO 2005b). In Tabelle 1 sind die von der WHO definierten sechs Phasen einer Pandemie sowie die gesundheitspolitischen Ziele dargestellt. Experten der WHO klassifizieren die augenblickliche Situation als Teil der Phase 3 (Juckett 2006).

Da eine zeitliche und räumliche Vorhersage der nächsten Pandemie nicht möglich ist, gilt die Aufforderung an alle Staaten, eigene nationale PandemiePläne in Anlehnung an den PandemiePlan der WHO zu entwickeln (WHO 2005a).

Die nationalen Pandemie-Pläne sollten fünf verschiedene von der WHO definierte Bereiche abdecken: Planung und Koordination von Aktionen, Überwachung und Feststellung neuer Fälle, Prävention und Eindämmung von Infektionen (nichtpharmazeutische Strategien, Impfungen, antivirale Medikamente), Verteilung der Aufgaben der verschiedenen Teile des Gesundheitssystems und Kommunikation mit der Bevölkerung (WHO 2005a).

Diese fünf Bereiche sollen am Beispiel der Phase 3 (die derzeitige Situation) im Folgenden näher beschrieben werden:

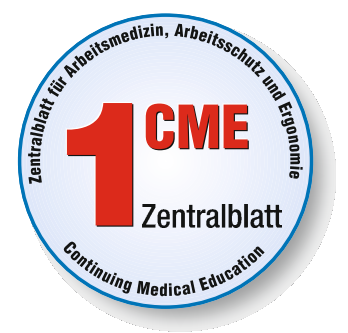


1. Planung und Koordination: Die WHO gibt betroffenen Staaten Hilfestellung, menschliche Fälle von H5N1Infektionen rasch $\mathrm{zu}$ erkennen und entsprechende Maßnahmen einzuleiten. Betroffene Staaten sollten sicherstellen, dass eine adäquate Reaktion möglich ist und durchgeführt wird.

2. Überwachung: Die WHO koordiniert die weltweite Feststellung neuer Fälle von humanen H5N1-Infektionen. Betroffene Staaten sollten dafür sorgen, dass eine weitere Verbreitung unter Menschen eingedämmt wird und Risikofaktoren identifizieren, die zu Infektion oder Übertragung führen können.

3. Prävention und Eindämmung von Infektionen: Die WHO stellt Daten über den Nutzen und die Durchführung der verschiedenen Strategien zur Verfügung. Außerdem treibt die WHO die Entwicklung eines Impfstoffes voran. Betroffene Staaten sollten die Übertragung von Mensch zu Mensch eindämmen und versuchen die Mortalität vorhandener Fälle zu senken.

4. Aufgaben des Gesundheitssystems: Die WHO stellt Leitlinien für die Krankenhausbehandlung Erkrankter zur Verfügung, ebenso für die Kontrolle der Infektionsübertragung. Betroffene Staaten sollten Maßnahmen ergreifen, um nosokomiale Übertragung auszuschließen und sicherstellen, dass Beschäftigte im Gesundheitssektor eine geschärfte Wahrnehmung für die Gefahren der Vogelgrippe beim Menschen haben.

5. Kommunikation: Sowohl die WHO als auch betroffene Staaten sollten sicherstellen, dass vorhandene Informationen transparent an die Öffentlichkeit kommuniziert werden (WHO 2005a).

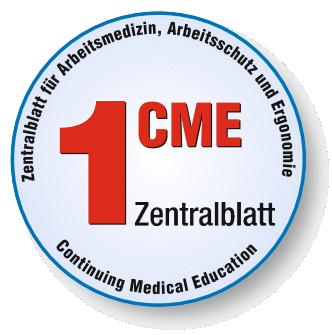

Tabelle 1: WHO-Klassifikation der Pandemie-Phasen (WHO 2005a)

Table 1: WHO-classification of pandemic stages (WHO 2005a)

Phasen

Gesundheitspolitische Ziele

\section{Interpandemischer Zeitraum}

Phase 1. Kein Nachweis neuer Influenzavirus-Subtypen beim Menschen. Ein Subtyp, der zu einem früheren Zeitpunkt Infektionen beim Menschen verursacht hatte, ist möglicherweise bei Tieren in Umlauf. Das Risiko menschlicher Infektionen wird als niedrig eingestuft.

Phase 2. Kein Nachweis neuer Influenzavirus-Subtypen beim Menschen. Bei Tieren in Umlauf befindliche Influenzaviren stellen jedoch ein erhebliches Risiko für Erkrankungen beim Menschen dar.
Die Vorbereitungen auf eine InfluenzaPandemie sollten global, regional, national und auf subnationaler Ebene vorangetrieben werden.

Das Risiko einer Übertragung auf Menschen sollte minimiert, mögliche Übertragungen sollten schnell aufgedeckt und gemeldet werden.

\section{Pandemie-Warnzeitraum}

Phase 3. Menschliche Infektion(en) mit einem neuen Subtyp, aber keine Ausbreitung von Mensch zu Mensch oder nur in extrem seltenen Fällen bei engem Kontakt.

Phase 4. Kleine Cluster mit begrenzter Übertragung von Mensch zu Mensch. Die räumliche Ausbreitung ist noch sehr begrenzt, so dass von einer unvollständigen Anpassung des Virus an den Menschen ausgegangen werden kann.

Phase 5. Große Cluster, die Ausbreitung von Mensch zu Mensch ist jedoch weiterhin örtlich begrenzt; es muss davon ausgegangen werden, dass das Virus besser an den Menschen angepasst ist, möglicherweise jedoch nicht optimal übertragbar ist (erhebliches Pandemierisiko).
Eine schnelle Charakterisierung neuer Virus-Subtypen wie auch der frühe Nachweis, die Meldung und Reaktion auf weitere Fälle sollten sichergestellt sein.

Das neue Virus sollte innerhalb eines umschriebenen Herdes eingedämmt werden, oder seine Ausbreitung sollte verzögert werden, um Zeit für vorbereitende Maßnahmen einschließlich der Entwicklung von Impfstoffen zu gewinnen.

Die Bemühungen, die Verbreitung des Virus einzudämmen oder zu verlangsamen, sollten maximiert werden, um eine Pandemie möglichst noch zu verhindern bzw. um Zeit für vorbereitende Maßnahmen zu gewinnen.

\section{Pandemie}

Phase 6. Pandemiephase: Zunehmende und anhaltende Übertragung in der Allgemeinbevölkerung.

Eine der Kernfragen ist die Behandlung bzw. die Prävention von H5N1Infektionen bei Menschen. Es wurden übereinstimmend drei unterschiedliche Interventionsmöglichkeiten benannt: Impfungen, antivirale Medikamente und Isolationsmaßnahmen bzw. Erschwerung der Verbreitung in der Bevölkerung (Bartlett 2006; Wicker et al. 2007). Impfungen wurden von der WHO als die bestmögliche präventive Maßnahme deklariert (WHO 2005a). Jedoch beinhaltet die Entwicklung eines wirksamen Impfstoffes einige Schwierigkeiten. Das H5-Virus ist tödlich für Hühnerembryos, die normalerweise verwendet werden um Viren für die Produktion von Influen-
Minimierung der Auswirkungen der Pandemie.

za-Impfstoffen zu züchten (Stephanson 2006). Außerdem ist möglicherweise ein gegen die jetzt zirkulierende Variante von $\mathrm{H} 5 \mathrm{~N} 1$ gerichteter Impfstoff gegen die mutierte, Pandemie auslösende Variante wirkungslos (Stephanson 2006). Zurzeit werden neue Methoden zur Impfstoffentwicklung erprobt, um sowohl die Quantität als auch die Qualität zu steigern (Bartlett 2006). Hierzu zählt u.a. die Vermehrung des möglichen Impfvirus auf Hühnerembryonen-unabhängigen Zellkulturen. Ein erster (Pandemie)Impfstoff unter Verwendung von Influenza A/H5N1 (A/Vietnam/1194/2004) wurde von der Europäische Arzneimittelagentur (European Medicines Agency - 
EMEA) im Frühjahr 2007 für die 27 EUStaaten zugelassen. Der darin enthaltene Virusstamm kann im Falle einer Pandemie durch den tatsächlich zirkulierenden Stamm ausgetauscht werden. Momentan ist jedoch mit einer Zeitspanne von etwa drei bis vier Monaten zwischen der Identifizierung des Pandemie-auslösenden Virus und dem Vorhandensein der ersten spezifischen Impfstoffe $\mathrm{zu}$ rechnen. Somit wäre die Initialphase der Pandemie weitgehend vorüber, bevor die ersten Personen geimpft werden könnten (Ferguson et al. 2006). In diesem Zusammenhang muss darauf hingewiesen werden, dass Pandemie-Pläne, die sich zu sehr auf das Vorhandensein eines Impfstoffes verlassen, nicht ausreichend sind (Shortridge 2006). Im Gegensatz zum Impfstoff sind antivirale Medikamente schon jetzt erhältlich. Die aktuellen H5N1-Viren sind resistent gegenüber den M2-Blockern Amantadin und Rimantadin (Allwinn \& Doerr 2005). Diese haben somit keine oder kaum Bedeutung bei der Bekämpfung und Prävention der Vogelgrippe. Wirksam ist jedoch die Substanzklasse der Neuraminidase-Inhibitoren Oseltamivir und Zanamivir (Allwinn \& Doerr 2005). Ihre Wirksamkeit ist jedoch nur dann gegeben, wenn sie spätestens 48 Stunden nach Beginn der Erkrankung verabreicht werden (Juckett 2006). Das bedeutet, dass eine prophylaktische Behandlung von Gefährdeten (z.B. Mitarbeiter im Gesundheitswesen mit Kontakt zu bestätigten oder verdächtigen Influenzafällen) erforderlich sein kann (Ferguson 2005) - doch wird dies kontrovers diskutiert (Moscona 2005; Wicker et al. 2007). Für die prophylaktische Einnahme von Neuramidase-Hemmern während der gesamten Pandemiephase besteht zudem keine Zulassung (längste zugelassene Verabreichung: 42 Tage) (Wicker et al. 2007). Darüber hinaus wurde mehrfach über Fälle berichtet, bei denen eine Resistenz gegenüber Oseltamivir vorlag. Diese waren gleichwohl alle sensitiv gegenüber Zanamivir (Bartlett 2006). Positiv zu bewerten ist außerdem, dass Oseltamivir zehn Jahre lang haltbar ist. Eine dritte entscheidende Interventionsmaßnahme liegt im Bereich der Bekämpfung der schnellen Ausbreitung des Virus. Hierbei geht es primär um Maßnahmen der sogenannten ,sozialen Distanz". Nach einer Analyse von Daten der Pandemie von 1918/19 geht man davon aus, dass ein Drittel der Übertragung über Haushaltskontakte, ein Drittel in Schulen und an Arbeitsplätzen und das letzte Drittel über sonstige soziale Kontakte erfolgt. Es wird angenommen, dass das größte Infektionsrisiko bei einer Pandemie eine erkrankte Person im eigenen Haushalt darstellt (Ferguson et al. 2006). Schulschließungen und Fernbleiben vom Arbeitsplatz sollten daher in nationalen Pandemie-Plänen bedacht werden. Hierbei sollte auch darauf eingegangen werden, was dies für die Wirtschaftslage bedeuten könnte und wie essentielle Dienstleistungen aufrechterhalten werden können. Eine effektive Eindämmungsstrategie sollte gezielte antivirale Behandlung von Erkrankten, sowie in Abhängigkeit vom Ausmaß der Pandemie das Tragen von Schutzmasken, ggf. das Aufstellen von physikalischen Barrieren, die den direkten Personenkontakt reduzieren (z.B. Plexiglaswände bei Kundenkontakten), bei besonders gefährdeten Personen die zeitlich begrenzte prophylaktische Behandlung und ggf. die Schließung von Schulen, öffentlichen Einrichtungen und eventuell Arbeitsplätzen beinhalten (Bartlett 2006; Ferguson et al. 2005; U.S. Department of Labor 2007; Wicker et al. 2007). Wie wirkungsvoll die Unterbindung von Infektionsübertragungen durch Atemmasken innerhalb der Allgemeinbevölkerung sein kann, zeigt eine Studie aus Hong Kong am Beispiel von SARS. Durch das Tragen von Atemmasken - selbst einfacher OP-Masken wurde gleichzeitig die Verbreitung des SARS-Virus durch infizierte Personen unterbunden und die Transmission auf noch nicht infizierte Personen reduziert (Lo 2005). Selbst wenn die Virusübertragung nicht vollständig unterbunden werden kann, ist es doch möglich den Pandemiekurvenverlauf nachhaltig $\mathrm{zu}$ verbessern (Wicker et al. 2007).

Einige der erforderlichen Interventionen setzen Verfassungsrechte und Menschenrechte außer Kraft, dies betrifft besonders den Bereich der Überwachung und Meldung neuer Fälle (WHO 2005b).
Die WHO fordert deswegen eine rechtlich gut fundierte und öffentlich kommunizierte Basis für solche Maßnahmen. Überwachung und Meldung neuer Fälle kann nur effektiv funktionieren, wenn alle Beteiligten über die bestehenden Pflichten umfassend informiert sind (Barnitz et al. 2006). Beispielsweise müssen Ärzte wissen, dass sie aufgrund der Meldepflicht an diesem Punkt von der ärztlichen Schweigepflicht entbunden sind.

Fazit: Die WHO hat eine Koordinationsfunktion inne, sie stellt Anleitungen sowie Hilfestellungen zur Verfügung, auf deren Basis die einzelnen Staaten ihre eigenen Maßnahmenpläne entwerfen sollten.

Zur globalen Situation muss leider gesagt werden, dass die Weltgemeinschaft momentan schlecht auf eine Pandemie vorbereitet wäre (Mermel 2005). Der Stand der Vorbereitungen und Planungen in den einzelnen Staaten ist jedoch sehr unterschiedlich (Coker \& MounierJack 2006).

\section{Situation in der asiatisch-pazifischen Region}

Die WHO hat die asiatisch-pazifische Region als potenzielles Epizentrum für neue endemische Erkrankungen, wie das Schwere Akute Respiratorische Syndrom (SARS) und die Vogelgrippe identifiziert (Coker \& Mounier-Jack 2006).

Seit den ersten Ausbrüchen der Vogelgrippe in Hong Kong im Jahre 2003 sind mehrere asiatische Staaten betroffen (Coker \& Mounier-Jack 2006). Der Stand der Vorbereitungen für eine Pandemie ist in den einzelnen Ländern der Region sehr unterschiedlich. Coker und Mounier-Jack kommen in ihrer Analyse der Situation in der asiatisch-pazifi-

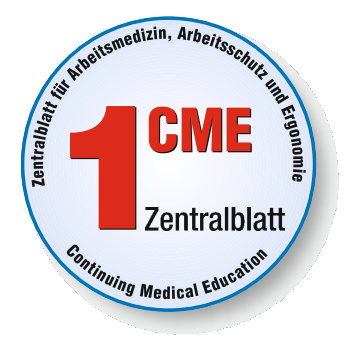


schen Region zu dem Ergebnis, dass einige Pandemie-Pläne mit den besten europäischen Planungen vergleichbar sind. Hierbei sind vor allem Hong Kong, Australien und Neuseeland zu nennen. Thailand, China und Vietnam haben bis jetzt nur Pläne publiziert, um die notwendigen Strategien überhaupt erst einmal zu entwickeln. Andere Länder haben ihre Pläne noch nicht fertig gestellt oder zumindest noch nicht veröffentlicht (Coker \& Mounier-Jack 2006).

Alle Staaten konzentrieren sich auf ein Szenario, in dem der Ausbruch in einer ländlichen Gegend erfolgen würde und betonen frühe Eindämmungsmaßnahmen. Ebenfalls koordinieren alle die Überwachungs- und Reaktionsstrategien für tierische und menschliche Fälle (Coker \& Mounier-Jack 2006). Dies ist von Bedeutung, da es in von der Vogelgrippe betroffenen Staaten wichtig ist, mit landwirtschaftlichen Interventionen dafür zu sorgen, dass die Zahl der infizierten Vögel reduziert wird (Bartlett 2006). Sofortiges Schlachten und Entsorgen des infizierten Tierbestandes hat den größten Effekt bei der Reduzierung von Neuinfektionen (Le Menach et al. 2006). Hierbei ist jedoch zu beachten, dass dieses Verfahren große finanzielle und soziale Auswirkungen für die betroffenen Geflügelbauern und das gesamte Land haben kann (Perez et al. 2005).

Weiterhin haben fast alle Staaten Strategien entwickelt, um die Bevölkerung $\mathrm{zu}$ informieren und über richtige Verhaltensweisen aufzuklären. Außerdem empfehlen alle Pläne Reiserestriktionen bzw. entsprechende Kontrollen (Coker \& Mounier-Jack 2006).

Entscheidende Schwächen in den Plänen wiesen Coker und Mounier-Jack auf folgenden Gebieten nach:

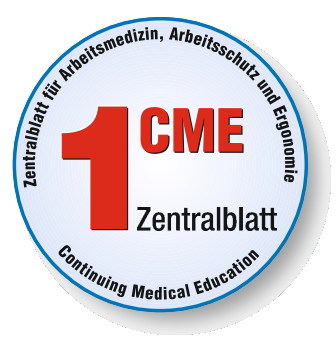

Tabelle 2: Verschiedene Komponenten nationaler Influenza-Programme in der Region Europa im März 2005 (Ciotti et al. 2005)

Table 2: Different components of national Influenza programs in March 2005 in the region of Europe (Ciotti et al. 2005)

\begin{tabular}{|c|c|c|c|c|c|c|}
\hline $\begin{array}{c}\text { Komponenten der } \\
\text { Influenza-Programme }\end{array}$ & \multicolumn{2}{|c|}{$\begin{array}{l}\text { Alle Länder } \\
\text { (56) }\end{array}$} & \multicolumn{2}{|c|}{$\begin{array}{l}\text { EU Staaten } \\
\text { (25) }\end{array}$} & \multicolumn{2}{|c|}{$\begin{array}{l}\text { Nicht-EU- } \\
\text { Staaten (31) }\end{array}$} \\
\hline $\begin{array}{l}\text { Existiert ein Überwachungs- } \\
\text { system für Influenza? }\end{array}$ & (55) & $98 \%$ & (25) & $100 \%$ & (30) & $97 \%$ \\
\hline $\begin{array}{l}\text { Gibt es ein Impfprogramm für } \\
\text { Risikogruppen? }\end{array}$ & (49) & $88 \%$ & (25) & $100 \%$ & (24) & $77 \%$ \\
\hline $\begin{array}{c}\text { Werden Influenza-Impfstoffe } \\
\text { kostenlos an Risikopatienten } \\
\text { abgegeben? }\end{array}$ & (35) & $63 \%$ & (18) & $72 \%$ & (17) & $55 \%$ \\
\hline $\begin{array}{l}\text { Wurde ein Vorrat an antivira- } \\
\text { len Medikamenten angelegt? }\end{array}$ & (24) & $43 \%$ & (13) & $52 \%$ & (11) & $36 \%$ \\
\hline $\begin{array}{l}\text { Existieren Laborkapazitäten } \\
\text { für die Diagnose einer } \\
\text { Influenza-Infektion? }\end{array}$ & (45) & $80 \%$ & (24) & $96 \%$ & (21) & $68 \%$ \\
\hline
\end{tabular}

- In vielen Plänen existiert keine konkrete Strategie zur Medikamentenverteilung.

- Die meisten Staaten sehen zu geringe Mengen an Medikamentenvorräten vor und wären somit nur in der Lage, einen kleinen Teil ihrer Bevölkerung zu versorgen.

- Die Impfstrategien der meisten Länder sind unzureichend (Coker \& MounierJack 2006). Hierzu ist zu bemerken, dass die meisten Staaten dieser Region keine oder wenig Möglichkeiten haben werden, ausreichend Zugang zu Medikamenten- und Impfstoffvorräten sicherzustellen (Coker \& MounierJack 2006).

Zur Situation in der asiatisch-pazifischen Region ist festzustellen, dass insbesondere in Ländern, die sich in sozial, ökonomisch oder politisch schwierigen Situationen befinden, die Entwicklung von Pandemieplänen keine hohe Priorität hat (Perez et al. 2005). Viele dieser Länder haben Probleme, die grundlegenden Anforderungen an ihre Gesundheitssysteme zu erfüllen (Juckett 2006). Einige Länder scheinen gut vorbereitet $\mathrm{zu}$ sein, andere haben noch einen weiten Weg zurückzulegen, bis sie ausreichend auf eine Pandemie vorbereitet sind. Anzumerken ist, dass alle Staaten dieser Region Maßnahmen der Reduzierung sozialer Kontakte priori- sieren und somit Wert auf Verminderung der Übertragungsrate legen. Dies mag daran liegen, dass sie aufgrund ihrer Situation weniger Möglichkeiten im Bereich der medikamentösen Therapie (Coker \& Mounier-Jack 2006).

\section{Situation in Europa}

Seit dem Erstnachweis in der europäischen Region im Oktober 2005 wurden bisher in 25 Ländern Infektionen von Wildvögeln und / oder Hausgeflügelbeständen mit dem H5N1-Virus gemeldet - dazu gehören Albanien, Bulgarien, Bosnien, Dänemark, Deutschland, Frankreich, Griechenland, Großbritannien, Italien, Kroatien, Niederlande, Österreich, Polen, Rumänien, Russland, Schweden, Schweiz, Serbien/Montenegro, Slowenien, Slowakei, Spanien, Tschechien, Ukraine, Ungarn und die Türkei (WHO 2008a). Nach einer zunächst großräumigen Verbreitung im Frühjahr 2006 gingen die gemeldeten H5N1-Infektionen ab Mai 2006 deutlich zurück (Friedrich-Löffler-Institut 2006a). Viele europäische Länder haben detaillierte Pandemie-Pläne entwickelt. Jedoch gibt es auch in diesen Regionen noch Unzulänglichkeiten beim Vorgehen im Pandemiefall und es existieren bedeutende Unterschiede zwischen den einzelnen Ländern (Mounier-Jack \& Coker und Entwicklung von Impfstoffen sahen 
2006). Seitdem die Vogelgrippe im Oktober 2005 Europa erreicht hat, sind deutlich mehr Anstrengungen unternommen worden, sich auf eine mögliche Pandemie vorzubereiten (Horstick et al. 2005). Zur WHO Region Europa gehören 56 Staaten, deren Situation recht unterschiedlich ist. Im Oktober 2005 hatten 46 der 56 Staaten einen Pandemie-Plan entwickelt (Horstick et al. 2005). Tabelle 2 gibt einen Überblick über das Vorhandensein nationaler InfluenzaProgramme, die auch schon jetzt in der Pandemie-Warnphase greifen.

Bei den Pandemie-Plänen fiel auf, dass osteuropäische Länder sich mehr auf traditionelle, nicht-pharmazeutische Maßnahmen zur Infektionskontrolle verließen, wohingegen westeuropäische Länder mehr auf den Einsatz von Impfstoffen und antiviralen Medikamenten setzen (Horstick et al. 2005). Obwohl davon ausgegangen werden kann, dass der Stand der Vorbereitungen in der europäischen Region gut ist, gibt es einige Bereiche, in denen Verbesserungen nötig sind. Zwar sahen alle Länder eine Notwendigkeit, mit WHO und EU-Institutionen zu kommunizieren, jedoch nur sieben Pläne sprachen die Notwendigkeit an, mit direkten Nachbarstaaten zusammen zu arbeiten (Mounier-Jack \& Coker 2006). Nirgends wurden Maßnahmen des Gesundheitswesens ausreichend mit veterinärmedizinischen Maßnahmen koordiniert (Mounier-Jack \& Coker 2006). Die Aufrechterhaltung von essentiellen Dienstleistungen während einer Pandemie wurde nicht genügend bedacht, ebenso sind wahrscheinlich die Strategien zur Umsetzung der Maßnahmen nicht immer angemessen (Mounier-Jack \& Coker 2006).

In Europa sind die notwendigen Ressourcen vorhanden, um sich auf eine Pandemie effizient vorzubereiten (MounierJack \& Coker 2006). Das Engagement der verantwortlichen Institutionen ist in den meisten Ländern hoch. Der Stand der Vorbereitungen ist als gut zu beurteilen, Überwachungs- und Erkennungsstrategien sind ausreichend entwickelt und mit genügend Laborkapazitäten koordiniert. Die Infrastruktur der Gesundheitssysteme ist stabil (MounierJack \& Coker 2006). Besonders auf dem
Gebiet der Koordination und Zusammenarbeit zwischen einzelnen Staaten müssen noch Fortschritte gemacht werden. Hier sollte auch die EU ihre Rolle als Koordinationspartner wahrnehmen (Mounier-Jack \& Coker 2006).

\section{Situation in Deutschland}

Erste Fälle der Vogelgrippe traten in Deutschland 2006 auf. Das Virus wurde primär in Wildvögeln nachgewiesen, befiel jedoch auch Nutzgeflügel. Ebenso wurden Infektionen bei einigen Säugetieren beobachtet. Es kam jedoch nicht $\mathrm{zu}$ einer Übertragung auf den Mensch. Seit Mai 2006 ist eine deutliche Abnahme von H5N1-Fällen zu beobachten (Friedrich-Löffler-Institut 2006a). Das Robert-Koch-Institut hat bestätigt, dass „das Auftreten menschlicher Fälle von aviärer Influenza durch A/H5N1-Viren in Deutschland prinzipiell möglich ist" (Friedrich-Löffler-Institut 2006b)

Im Bereich Geflügelhaltung existieren folgende Regelungen in Deutschland: Präventiv wurde ein Aufstallungsgebot verordnet, es kam zur Festlegung von Risikogebieten mit Aufstallungsgebot (Friedrich-Löffler-Institut 2006a). Auf der Basis der derzeit verfügbaren veterinärmedizinischen Impfstoffe und Nachweissysteme und der sorgfältigen Abwägung der Vor- und Nachteile wird eine Schutzimpfung von Nutzgeflügel derzeit nicht empfohlen (FriedrichLöffler-Institut 2006a). Die Vögel scheiden das Virus mit dem Kot aus, so dass die Inhalation kontaminierter Staubpartikel oder mangelnde Händehygiene vermutlich die hauptsächlichen Übertragungswege auf den Menschen darstellen. Als Schutzmaßnahme wird empfohlen, den Kontakt mit infiziertem Geflügel oder infizierten Tieren zu vermeiden und wo dies unumgänglich ist, einen adäquaten Atemschutz zu tragen und für eine ausreichende Desinfektion aller Kontaktstellen zu sorgen (Bundesanstalt für Arbeitsschutz und Arbeitsmedizin 2005).

Deutschland besitzt einen Influenza Pandemie-Plan, der in einer Analyse von Mounier-Jack und Coker einen 80\% Vollständigkeitsgrad erreichte (MounierJack \& Coker 2006). Im Bereich Planung und Koordination erreichte der deutsche Plan 60\%, im Bereich Überwachung $80 \%$ Vollständigkeit. Für den Bereich Interventionen des Gesundheitssystems erzielte er $70 \%$, für die Aufrechterhaltung von essentiellen Dienstleistungen $30 \%$, für Kommunikation mit der Öffentlichkeit $60 \%$ und für den Testbereich, wie die Pläne umgesetzt werden, erreichte der deutsche Plan 70\% Vollständigkeit (Mounier-Jack \& Coker 2006). Damit gehört Deutschland zu den führenden Ländern bei der Vorbereitung auf eine Pandemie. Es sind weit reichende Vorbereitungen für eine mögliche Pandemie getroffen worden. Deutschland hat die nötige Infrastruktur um auf eine Pandemie bestmöglich zu reagieren. Jedoch sind auch hier noch Verbesserungen notwendig.

\section{Zusammenfassung}

Die meisten Staaten scheinen die Gefahr durch das Influenza-A-Virus H5N1 und eine mögliche Influenza-Pandemie erkannt zu haben. Die Reaktionen auf diese Erkenntnis und ebenso der Stand der Vorbereitungen sind jedoch sehr unterschiedlich. Hierbei spielt nicht selten die ökonomische und politische Situation der jeweiligen Staaten eine entscheidende Rolle. Der europäische Raum gehört zu den mit am besten vorbereiteten Regionen. Auch mit allen existierenden Maßnahmen und mit den noch zu entwickelnden Strategien wird es jedoch wahrscheinlich nicht möglich sein, eine Pandemie zu verhindern. Ziel sollte daher sein, die Auswirkungen zu minimieren. Die Vorbereitungen für eine mögliche Pandemie sind keineswegs abgeschlossen. Nicht einmal Länder wie Deutschland mit exzellenten Ressourcen haben alle Vorgaben der WHO erfüllen können. Es besteht großer Bedarf an Forschung hinsichtlich antiviraler Medi-

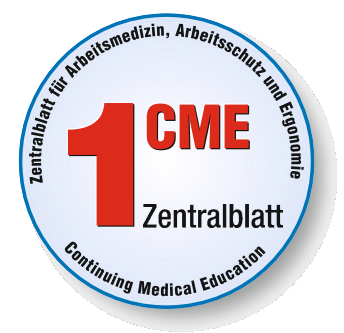


kamente und zur Impfstoffentwicklung. Gleichzeitig sollte die Nutzung bewährter Strategien der Verhinderung von Übertragungen (z.B. durch das Tragen von Atemschutzmasken, Erziehung zur verbesserten und konsequenten (Hände) Hygiene, Nutzung von physikalischen Barrieren, Möglichkeiten sozialer Distanzierung) nicht vergessen werden.

Die Weltgemeinschaft sollte sich darüber Gedanken machen, wie Entwicklungs- und Schwellenländer Zugang zu Medikamenten und Impfstoffen erhalten können. Dies gilt besonders für die Staaten in der asiatisch-pazifischen Region, die möglicherweise als erste und am heftigsten von einer H5N1-Pandemie betroffen wäre.

Niemand weiß, wann die nächste Pandemie auftreten wird, jedoch ist bereits jetzt deutlich, dass es sich auch um eine Herausforderung in Sachen globaler Solidarität handeln wird.

\section{Literatur}

Allwinn R, Doerr HW (2005) Wie gefährlich ist die Vogelgrippe für den Menschen. Mediz Klinik 100:710-713

Barnitz L, Berkwitz M (2006) The Health care response to Pandemic Influenza. Ann Intern Med 145:135-137

Bartlett JG (2006) Planning for Avian Influenza. Ann Intern Med 145:141-144

Bundesanstalt für Arbeitsschutz und Arbeitsmedizin (2005) Beschluss 609: Arbeitschutz beim Auftreten von Influenza unter besonderer Berücksichtigung des Atemschutzes. http://www.baua.de/de/Themen-von-A-Z Biologische-Arbeitsstoffe/TRBA Beschluss-609.html (Letzter Zugriff am 17. April 2008)
Ciotti M, Karcher F, Ganter B, Tüll P (2005) Results of survey of national pandemic preparedness in Europe. Euro Surveill 10(3):E050303.1

Coker R, Mounier-Jack S (2006) Pandemic influenza preparedness in the Asia-Pacific region. Lancet 368:886-889

Ferguson NM, Cummings D, Cauchemez S (2005) Strategies for containing an emerging influenza pandemic in Southeast Asia. Nature 437:209-214

Ferguson NM, Cummings D, Fraser C, Cajka J, Cooley P, Burke D (2006) Strategies for mitigating an influenza pandemic. Nature 442:448-452

Friedrich-Löffler-Institut (2006a) Bewertung des Risikos zur Einschleppung von hochpathogenem aviärem H5N1-Virus in Hausgeflügelbestände in Deutschland. Bundesforschungsinstitut für Tiergesundheit.

http://www.fli.bund.de/1191.htm

(Letzter Zugriff am 17. April 2008)

Friedrich-Löffler-Institut (2006b) Presseinformation vom 7. September 2006: Hohes Risiko für Nutzgeflügel durch „Vogelgrippe“. Bundesforschungsinstitut für Tiergesundheit.

http://www fli.bund de/1191 htm (Letzter Zugriff am 17. April 2008)

Horstick O, Kaiser R, Ciotti M (2005) Europe makes progress in preparing for influenza pandemic, but further work needed. Euro Surveill 10(11):E051117.1

Juckett G (2006) Avian Influenza: Preparing for a Pandemic. Am Fam Phys 74:783-790

Le Menach A, Vergu E, Grais RF, Smith DL, Flahault A (2006) Key strategies for reducing spread of avian influenza among commercial poultry holdings: lessons for transmission to humans. Royal Society 273:2467-2475

Lo JY, Tsang TH, Leung YH, Yeung EY, Wu T, Lim WW. (2005) Respiratory Infections during SARS Outbreak, Hong Kong, 2003. Emerg Infect Dis (11):1738-1741

Mermel LA (2005) Pandemic avian influenza. Lancet 5:666-667

Moscona A (2005) Neuraminidase inhibitors for influenza. N Engl J Med 353(13):1363-1373

Mounier-Jack S, Coker R (2006) How prepared is Europe for pandemic influenza? Analysis of national plans. Lancet 367:1405-1411
Normile D (2006) WHO proposes plan to stop Pandemic in its tracks. Science 311:315-316

Perez DR, Sorell EM, Donis RO (2005) Avian Influenza: an omnipresent pandemic threat. Pediatr Infect Dis J 24:208-216

Shortridge KF (2006) Influenza pandemic preparedness: gauging from EU plans. Lancet 367:1374-1375

Stephenson I (2006) H5N1 Vaccines: how prepared are we for a pandemic? Lancet 368:965-966

U.S. Department of Labor (2007) Guidance on Preparing Workplaces for an Influenza Pandemic. OSHA 3327-02N 2007.

http://www.osha.gov/Publications/

OSHA3327pandemic.pdf

(letzter Zugriff am 17. April 2008)

Wicker S, Dickmann P, Rabenau HF, Gottschalk R (2007) Influenzapandemieplanung: Eine Herausforderung für Unternehmen, Arbeitsmedizin und öffentlichen Gesundheitsdienst. Zbl Arbeitsmedizin 57:202-10

Wong S, Yuen K (2006) Avian Influenza Virus Infections in Humans. Chest 129:156-168

World Health Organization (WHO 2005a) Global influenza preparedness plan. Department of Communicable Disease Surveillance and Response, Global Influenza Programme. http://www.who.int/csr/resources/publications/ influenza

(Letzter Zugriff am 17. April 2008)

World Health Organization (WHO) (2005b) Checklist for influenza pandemic preparedness planning. Department of Communicable Disease Surveillance and Response, Global Influenza Programme.

http://www.who.int/csr/resources/publications/ influenza

(Letzter Zugriff am 17. April 2008)

World Health Organization (WHO 2008) http://www.who.int/csr/disease/avian_influenza/ country/cases table_2008_04_08/en/ index html

(letzter Zugriff am 17.April 2008)

World Health Organization (WHO 2008a) http://gamapserver.who.int/mapLibrary/Files/ Maps/Global_SubNat_H5N1inAnimal ConfirmedCUMMULATIVE_20080414.png (letzter Zugriff am 17.April 2008)

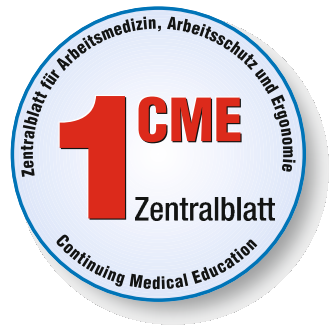




\section{CME-Fragen: Die Bedeutung der aviären Influenza für das Gesundheitssystem}

1. Welche Aussage zur Influenza trifft zu?

A Influenza-B-Viren kommen nur bei Geflügel vor.

B Die Influenza-Viren gehören zur Gruppe der Flaviviridae.

C Die Unterscheidung der Influenza-A-Viren in Subtypen erfolgt anhand der Oberflächenproteine Hämagglutinin und Neuraminidase.

D Das auslösende Agens der aviären Influenza sind Influenza-C-Viren.

E Die aviäre Influenza ist eine Viruskrankheit, die insbesondere Schweine und Rinder befällt.

2. Welche Aussage zu einer möglichen Influenza-Pandemie trifft zu?

A Die WHO definiert drei verschiedene Phasen einer Pandemie.

B Die WHO erstellt die nationalen Influenza-Pläne.

C Experten der WHO klassifizieren die augenblickliche Situation als Teil der Phase 1 einer Pandemie.

D Impfungen wurden von der WHO als die bestmögliche präventive Maßnahme beim Auftreten einer Influenza-Pandemie deklariert.

E Momentan ist jedoch mit einer Zeitspanne von etwa ein bis zwei Wochen zwischen dem Ausbruch einer Pandemie und dem Vorhandensein der ersten Impfstoffe zu rechnen.

3. Welche Aussage zu einer möglichen Influenza-Pandemie trifft nicht zu?

A Es wird angenommen, dass das größte Infektionsrisiko bei einer Pandemie eine erkrankte Person im eigenen Haushalt darstellt.

B Der Stand der Vorbereitungen und die Planungen in den einzelnen Staaten befinden sich zurzeit auf einem sehr ähnlichen Niveau.

C Schulschließungen und Fernbleiben vom Arbeitsplatz müssen in nationalen Pandemie-Plänen bedacht werden.

D Zur globalen Situation ist zu sagen, dass die Länder momentan insgesamt schlecht auf eine Pandemie vorbereitet wären.

E Ärzte sind aufgrund der Meldepflicht im Fall einer Influenza-Pandemie von der ärztlichen Schweigepflicht diesbezüglich entbunden.

4. Welche Aussage zur Eindämmung einer möglichen Influenza-Pandemie trifft zu?

A Im Gegensatz zum Pandemie-Impfstoff sind antivirale Medikamente schon jetzt erhältlich.

B Interventionsmaßnahmen wie soziale Distanz werden als wenig oder kaum effektiv bei der Virusausbreitung angesehen.

C Nicht wirksam sind Medikamente der Substanzklasse der Neuraminidase-Inhibitoren.

D Die aktuellen H5N1-Viren sind sensibel gegenüber den M2-Blockern Amantadin und Rimantadin.

E Antivirale Medikamente können bis zu zwei Wochen nach Beginn der Erkrankung wirksam appliziert werden.

5. Welche Aussage zu Influenza-Pandemie-Plänen trifft zu?

A Die Aufrechterhaltung bedeutsamer Dienstleistungen während einer Pandemie wird in den vorliegenden Pandemie-Plänen in der Regel sorgfältig bedacht.

B Maßnahmen des Gesundheitswesens werden in den vorliegenden Pandemie-Plänen exakt mit veterinärmedizinischen Maßnahmen koordiniert.

C Die europäischen Pandemie-Pläne weisen keine Lücken beim Vorgehen im Fall einer Pandemie auf.

D Bei den europäischen Pandemie-Plänen gibt es kaum Unterschiede in der Qualität der Pläne.

E Ende des Jahres 2005 lagen in über 80\% der europäischen Staaten Pandemie-Pläne vor. 


\section{Welche Aussage zu H5N1 und einer möglichen Influenza-Pandemie trifft nicht zu?}

A Das sofortige Schlachten von infizierten Tieren hat den größten Effekt bei der Reduzierung von Neuinfektionen z.B. bei Infektion von Hühnern.

B Die meisten Staaten haben ausreichende Mengen an Medikamentenvorräten zur Verteilung an die Bevölkerung.

C In vielen Plänen existiert keine ausreichend detaillierte Beschreibung einer Strategie zur Verteilung von Medikamenten.

D Thailand, China und Vietnam haben bisher nur wenig entwickelte Pläne für den Fall einer Pandemie publiziert.

E Hong Kong, Australien und Neuseeland weisen gut entwickelte Pandemie-Pläne vor.

\section{Welche Aussage zu einer möglichen Influenza-Pandemie trifft nicht zu?}

A Präventiv wurde in Deutschland ein Aufstallungsgebot verordnet.

B Derzeit wird eine Schutzimpfung gegen das H5N1-Virus von Nutzgeflügel in Deutschland nicht generell empfohlen.

C In Deutschland wurden Risikogebiete mit Aufstallungsgebot festgelegt.

D Als Schutzmaßnahme gegen das H5N1-Virus wird unter anderem eine ausreichende Desinfektion der Kontaktstellen empfohlen.

E Das Auftreten menschlicher Fälle von aviärer Influenza durch H5N1-Viren wird prinzipiell ausgeschlossen.

\section{Welche Aussage zu einer möglichen Influenza-Pandemie trifft nicht zu?}

A In Deutschland wurde das H5N1-Virus bereits bei Nutzgeflügel nachgewiesen.

B Bei der Koordination und Zusammenarbeit zwischen den einzelnen europäischen Staaten müssen bei den Vorbereitungen auf eine Pandemie noch Fortschritte gemacht werden.

C Es wurden bisher keine Infektionen bei Säugetieren beobachtet.

D Das H5N1-Virus wurde in Deutschland bereits in Wildvögeln nachgewiesen.

E Im Bereich der Geflügelhaltung existieren in Deutschland spezielle Regelungen.

\section{Welche Aussage zum H5N1-Virus trifft nicht zu?}

A Die Übertragung des H5N1-Virus von Mensch zu Mensch ist bisher nicht sehr effektiv.

B Die Vogelgrippe wurde erstmals im 19. Jahrhundert in Italien beschrieben.

C Influenza-Viren gehören zur Gruppe der Orthomyxoviridae.

D Die Mortalitätsrate des H5N1-Virus ist beim Menschen gering.

E Das H5N1-Virus ist humanpathogen.

\section{Welche Aussage zur aviären Influenza trifft zu?}

A Deutschland benötigt bisher keinen Influenza Pandemie-Plan.

B Deutschland ist bisher nicht von der Vogelgrippe betroffen.

C Ein Atemschutz wird als nicht sinnvolle Schutzmaßnahme angesehen.

D Übertragungswege stellen insbesondere die Inhalation kontaminierter Staubpartikel oder mangelnde Händehygiene dar.

E Als Schutzmaßnahme wird derzeit ausschließlich empfohlen, den Kontakt mit infiziertem Geflügel oder infizierten Tieren zu vermeiden. 


\section{CME-Fragen: Antwortbogen Seite 1}

\section{A Angaben zur Person}

Name, Vorname, Titel:

Straße, Hausnummer:

PLZ / Ort:

Anschrift: $\square$ privat $\square$ dienstlich

EFN-Nummer:

Ich bin Mitglied der Ärztekammer (bitte Namen der Kammer eintragen):

Jahr meiner Approbation:

Ich befinde mich in der Weiterbildung zum:

Ich habe eine abgeschlossene Weiterbildung in (bitte Fach eintragen):

Ich bin tätig als: $\square$ Assistenzarzt $\square$ Oberarzt $\square$ Chefarzt $\square$ niedergelassener Arzt Sonstiges:

\section{B Lernerfolgskontrolle}

Bitte nur eine Antwort pro Frage ankreuzen

C Erklärung

\begin{tabular}{|c|c|c|c|c|}
\hline 1 & $\mathbf{A}$ & B & C & D \\
\hline 2 & A & B & C & D \\
\hline 3 & A & B & C & D \\
\hline 4 & A & B & C & D \\
\hline 5 & A & B & C & D \\
\hline
\end{tabular}

Ich versichere, dass ich die Beantwortung der Fragen selbst und ohne Hilfe durchgeführt habe
Unterschrift

E

Zertifizierungsfeld (wird durch den Verlag ausgefüllt)

Bitte in dieses Feld die CME-Wertmarke kleben oder Ihre Abonnement-Nummer eintragen: (siehe Adressaufkleber)

Ihr Ergebnis

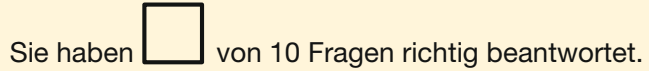

Sie haben

bestanden und 1 CME-Punkt erworben.

nicht bestanden

Heidelberg, den

Datum

Stempel/Unterschrift

Bitte unbedingt Rückseite ausfüllen! Bitte unbedingt Rückseite ausfüllen! Bitte unbedingt Rückseite ausfüllen! 


\section{CME-Fragen: Antwortbogen Seite 2}

\section{F $\quad$ Fragen zur \\ Zertifizierung}

Eine Antwort pro Frage. Bitte unbedingt ausfüllen bzw. ankreuzen, da die Evaluation sonst unvollständig ist!

\section{Didaktisch-methodische Evaluation}

\section{Das Fortbildungsthema kommt in meiner Tätigkeit als Ärztin/Arzt}

$\square$ häufig vor $\square$ selten vor

regelmäßig vor $\quad \square$ gar nicht vor

2 Durch die Fortbildung habe ich im Umgang mit der Thematik

eine feste Strategie entwickeln können

Ansätze einer Strategie entwickeln können

keine Strategie entwickeln können

\section{Hinsichtlich der Inhalte der Fortbildung}

$\square$ habe ich wesentliche neue Kenntnisse geboten bekommen

habe ich keine wesentlichen neue Kenntnisse geboten bekommen

4 Wurden aus der Sicht Ihrer täglichen Praxis heraus wichtige Aspekte des Themas

nicht erwähnt?

$\square$ ja welche:

nein

zu knapp behandelt?

ja welche:

nein

überbewertet?

ja welche:

\section{Verständlichkeit des Beitrags}

Der Beitrag ist nur für Spezialisten verständlich

Der Beitrag ist auch für Nicht-Spezialisten verständlich

\section{Beantwortung der Fragen}

Die Fragen lassen sich aus dem Studium des Beitrags allein beantworten

Die Fragen lassen sich nur unter Zuhilfenahme zusätzlicher Literatur beantworten

7 Die Aussagen des Beitrags benötigen eine ausführlichere Darstellung

zusätzlicher Daten

von Befunden bildgebender Verfahren

die Darstellung ist ausreichend

8 Wieviel Zeit haben Sie für das Lesen des Beitrages und die Bearbeitung des Quiz benötigt?

Bitte senden Sie den vollständigen Antwortbogen zusammen mit einem an Sie selbst adressierten und ausreichend frankierten Rückumschlag an den

Dr. Curt Haefner-Verlag $\square$ Stichwort: CME-Zentralblatt $\mathbf{\square}$ Dischingerstr. 8 @ 69123 Heidelberg
Einsendeschluss ist der 31. 10.2008 